\title{
Cranked Skyrme-Hartree-Fock-Bogoliubov approach for a mean-field description of nuclear rotations near the drip line
}

\author{
Kenichi Yoshida ${ }^{1, *}$ \\ ${ }^{1}$ Department of Physics, Kyoto University, Kyoto, 606-8502, Japan
}

(Dated: February 1, 2022)

\begin{abstract}
To describe the yrast states in weakly bound nuclei, I directly solve the coordinate-space cranked Skyrme-Hartree-Fock-Bogoliubov equation on a three-dimensional lattice with the continuum states discretized in a box. After the numerical demonstration for the ground-state band in a medium-mass nucleus, I apply the newly-developed method to neutron-rich even- $N \mathrm{Mg}$ isotopes. I find that the appearance of the significantly low $I^{\pi}=2^{+}$state in ${ }^{40} \mathrm{Mg}$ is mainly due to the suppression of pairing. The calculation predicts that the $2^{+}$state in ${ }^{42} \mathrm{Mg}$ appears as high in energy as in ${ }^{34-38} \mathrm{Mg}$ whereas the triaxial deformation is enhanced in non-zero spin states. The present numerical framework offers a practical approach for investigating the near yrast states systematically and revealing structures unique in drip-line nuclei.
\end{abstract}

\section{INTRODUCTION}

A diverse variety of modes of motion show up in nuclei. To explore unique structures in exotic nuclei, various spectroscopic studies have been carried out via decays and making use of direct reactions. Nuclear deformation is a collective phenomenon that has long attracted interest; a nucleus is deformed as it moves away from the magic number due to the many-body correlation. It has been turned out that the shell structure evolves as the conventional magic numbers disappear and new ones appear instead, depending on the combination of the particle numbers of protons and neutrons [1]. Energies of excited nuclear states are often among the first quantities accessible in experiments and have been used as an indicator of the changing shell structure and the onset of deformation [2].

Rotational motion is a manifestation of the spontaneous breaking of the rotational symmetry [3]. The cranking approximation provides an intuitive picture of the rotation of quantum systems, and the cranked shell model is a standard tool to investigate the microscopic structure of nuclear rotations near the ground state and at high spins [4-7]. The cranked shell model in the framework of the nuclear energy-density functional (EDF)the cranked Kohn-Sham (KS) approach-has provided a systematic and quantitative description of the (near) yrast states from light to heavy nuclei [8-10]. The accumulation of experimental data for low spins in neutronrich nuclei has stimulated the application of the cranked KS approach to the exploration of the rotational motions unique in weakly bound nuclei.

Since the moments of inertia of neutron-rich nuclei are sensitive to the pairing and weak binding [11], one needs to solve the cranked KS-Bogoliubov (KSB) equation in the coordinate space to describe properly the weak binding and the pairing embedded in the continuum [12], which is computationally demanding be-

\footnotetext{
* E-mail: kyoshida@ruby.scphys.kyoto-u.ac.jp
}

cause many symmetries are broken and the quasiparticle wave functions are spatially extended. The low-lying collective states have been investigated microscopically in beyond-mean-field approaches, such as the projected shell model [13], the generator-coordinate method and the collective Hamiltonian [8-10, 14]. Including the continuum effects in these approaches is much more demanding.

The island of inversion has been the subject of much experimental and theoretical interest [15]. A systematic calculation for the Mg isotopes in the mean-field approximation produces a spherical configuration in ${ }^{32} \mathrm{Mg}$, a soft potential energy surface in ${ }^{34} \mathrm{Mg}$, and a prolate configuration in ${ }^{36,38,40} \mathrm{Mg}$ for the ground state [16]. It has been clarified that the shape fluctuation and the correlation beyond the mean-field approximation is significant in ${ }^{32} \mathrm{Mg}$ [17-19]. Experimentally, the measurement of not only the low excitation energy of the first $I^{\pi}=2^{+}$state but the energy of the $4^{+}$state and their ratio with $R_{4 / 2}$ being greater than three has revealed a well-deformed structure in ${ }^{34,36,38} \mathrm{Mg}[20]$. A significantly low $2^{+}$state in ${ }^{40} \mathrm{Mg}$ is not reproduced by any theoretical models ${ }^{1}$, and it indicates a unique feature associated with the weak binding [24]. As a coherent contribution of the pairing in the continuum states induces an enhanced quadrupole transition to the low-lying vibrational state in the $\mathrm{Mg}$ isotopes [25-29], the roles of the weak binding and the continuum coupling in the rotational motions are interesting to study.

I investigate in this article the low-spin states in the neutron-rich $\mathrm{Mg}$ isotopes close to the drip line. Then, I try to clarify the roles of the weak binding and excess neutrons in the low-lying excited states. To this end,

\footnotetext{
${ }^{1}$ The configuration-mixing calculation using the Gogny force taking only the axial symmetry produces the increase in $E\left(2_{1}^{+}\right)$from $N=22$ to 26 contrary to the measurements and a drop in energy at $N=28[18,21]$. However, the inclusion of the triaxial deformation reproduces the isotopic dependence up to ${ }^{38} \mathrm{Mg}$, and the drop at ${ }^{40} \mathrm{Mg}$ is washed out. [22]. The relativistic approach in Ref. [23] produces a strong neutron-number dependence.
} 
I develop a new framework of the cranked shell model within the nuclear EDF approach, which is capable of handling nuclides with arbitrary mass numbers $[8,14]$.

\section{METHOD}

To describe the (near) yrast states with proper account of the pairing in the continuum states, I directly solve the coordinate-space cranked Skyrme-KSB or Hartree-FockBogoliubov equation in the quasiparticle basis:

$$
\begin{array}{r}
\sum_{\sigma^{\prime}}\left[\begin{array}{cc}
h_{\sigma \sigma^{\prime}}^{q \prime}(\boldsymbol{r}) & \tilde{h}_{\sigma \sigma^{\prime}}^{q}(\boldsymbol{r}) \\
4 \sigma \sigma^{\prime} \tilde{h}_{-\sigma-\sigma^{\prime}}^{q *}(\boldsymbol{r}) & -4 \sigma \sigma^{\prime} h_{-\sigma-\sigma^{\prime}}^{q *}(\boldsymbol{r})
\end{array}\right]\left[\begin{array}{l}
\varphi_{1, \alpha}^{q}\left(\boldsymbol{r} \sigma^{\prime}\right) \\
\varphi_{2, \alpha}^{q}\left(\boldsymbol{r} \sigma^{\prime}\right)
\end{array}\right] \\
=E_{\alpha}\left[\begin{array}{l}
\varphi_{1, \alpha}^{q}(\boldsymbol{r} \sigma) \\
\varphi_{2, \alpha}^{q}(\boldsymbol{r} \sigma)
\end{array}\right],
\end{array}
$$

which is obtained by extending the formalism developed for describing the ground-state properties of even-even nuclei near the drip line [12]. Here the single-particle Routhian and the pair Hamiltonian are defined by using a Skyrme EDF combined with a pairing functional $E\left[\rho, \tilde{\rho}, \tilde{\rho}^{*}\right]$ as $h_{\sigma \sigma^{\prime}}^{q \prime}(\boldsymbol{r})=\frac{\delta E\left[\rho, \tilde{\rho}, \tilde{\rho}^{*}\right]}{\delta \rho_{\sigma^{\prime} \sigma}^{q}(\boldsymbol{r})}-\left(\lambda^{q}+\omega_{\mathrm{rot}} j_{z}\right) \delta_{\sigma \sigma^{\prime}}$ and $\tilde{h}_{\sigma \sigma^{\prime}}^{q}(\boldsymbol{r})=\frac{\delta E\left[\rho, \tilde{\rho}, \tilde{\rho}^{*}\right]}{\delta \tilde{\rho}_{\sigma^{\prime} \sigma}^{*}(\boldsymbol{r})}$. I define the $z$-axis as a quantization axis of the intrinsic spin and consider the system rotating uniformly about the $z$-axis. I take the natural units: $\hbar=c=1$.

The Skyrme-KSB equation in the three-dimensional (3D) Cartesian-mesh has been solved by employing the contour integral technique and the shifted Krylov subspace method for the Green's function [30, 31] to circumvent the successive diagonalization of the matrix with huge dimension. Very recently, the direct diagonalization of the KSB Hamiltonian in the 3D Cartesian-mesh has been achieved [32]. The numerical procedure to solve Eq. (1) in the present study is basically the same as in solving the cranked KS equation in Refs. [33, 34]. I impose the reflection symmetry about the $(x, y)-,(y, z)$ - and $(z, x)$-planes to reduce the computational time. Thus, the parity $\mathfrak{p}_{k}(= \pm 1)$ and $z$-signature $r_{k}(= \pm \mathrm{i})$ are a good quantum number. I solve Eq. (1) by diagonalizing the KSB Hamiltonian directly in the 3D Cartesian-mesh representation with the box boundary condition. Thanks to the reflection symmetries, I have only to consider explicitly the octant region in space with $x \geq 0, y \geq 0$, and $z \geq 0$; see Refs. [35, 36] for details. I use a 3D lattice $\operatorname{mesh} x_{i}=i h-h / 2, y_{j}=j h-h / 2, z_{k}=k h-h / 2(i, j, k=$ $1,2, \cdots M)$ with a mesh size $h$. The dimension of the KSB Hamiltonian is thus $8 M^{3}$. To check the convergence of the results with respect to the box size and to investigate the effect of the weak binding, I change $h$ and $M$ in the discussion below. The differential operators are represented by the use of the 9-point formula of the finite difference method. For diagonalizing the matrix of Eq. (1), I use the ScaLAPACK PDSYEv subroutine [37]. A modified Broyden's method [38] is utilized to calculate new densities during the selfconsistent iteration. For

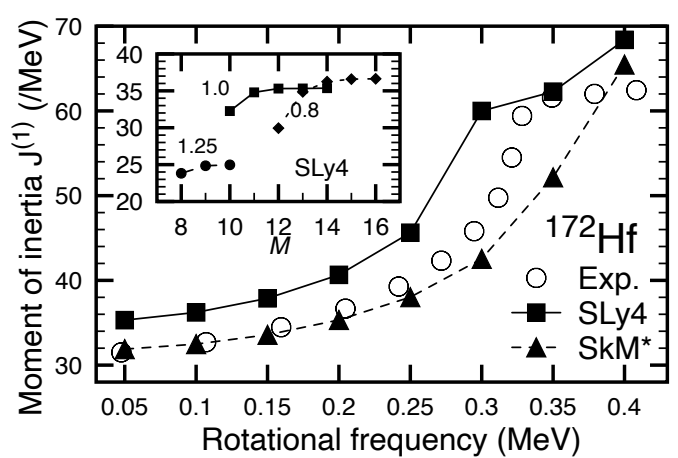

FIG. 1. Kinematic moments of inertia $\mathcal{J}^{(1)}$ as functions of the rotational frequency. The experimental data [39] are denoted by open symbols. Dependence of $\mathcal{J}^{(1)}$ evaluated at $\omega_{\text {rot }}=0.05 \mathrm{MeV}$ on the parameters $h$ and $M$ is shown in the inset.

each iteration, it took 13.0, 52.5, and 91.0 core-hours for $M=12,14$, and 16 , respectively at the Yukawa-21 computer facility. The quasiparticle energy is cut off at 60 $\mathrm{MeV}$, which has almost no significant change in computational time but determines the memory required during the calculation.

\section{RESULTS AND DISCUSSION}

\section{A. Ground-state band in ${ }^{172} \mathbf{H f}$}

To see the validity of the present framework, I perform the calculation for the ground-state rotational band in ${ }^{172} \mathrm{Hf}$ as a typical example of the collective rotation. Figure 1 shows the calculated kinematic moments of inertia $\mathcal{J}^{(1)}$ as functions of the rotational frequency. Here, $\mathcal{J}^{(1)}$ is defined by $J_{z} / \omega_{\text {rot }}$. I employed the SLy4 [40] and $\mathrm{SkM}^{*}$ [41] functionals together with the YamagamiShimizu-Nakatsukasa (YSN) pairing EDF in Ref. [42]. The inset shows the calculated $\mathcal{J}^{(1)}$ at $\omega_{\text {rot }}=0.05 \mathrm{MeV}$ obtained by varying $h$ and $M$. One sees the results are converged at about 11-12 fm with respect to the box size. The calculated rms radius is $5.37 \mathrm{fm}$ and $5.28 \mathrm{fm}$ for neutrons and protons, respectively. Thus, a rough estimate for a sufficient box size is that one needs a box about twice as large as the rms radius. Since the mesh size $h=1.0 \mathrm{fm}$ gives a reasonable convergence as found in the early studies [43, 44], I use $M=12$ and $h=1.0$ $\mathrm{fm}$ in the following. It is noticed that a systematic numerical investigation in Ref. [45] revealed that the 3D mesh calculation gives a remarkably high precision with apparently coarse meshes such as $h=1.0 \mathrm{fm}$.

The present model describes well the low spin states and the band crossing. Around $\omega_{\text {rot }}=0.25 \mathrm{MeV}$, the alignment of neutrons in the $i_{13 / 2}$ orbital occurs for the case of SLy 4 whereas this is lower than the measurement $\sim 0.3 \mathrm{MeV}$. One sees that the rotational property beyond 


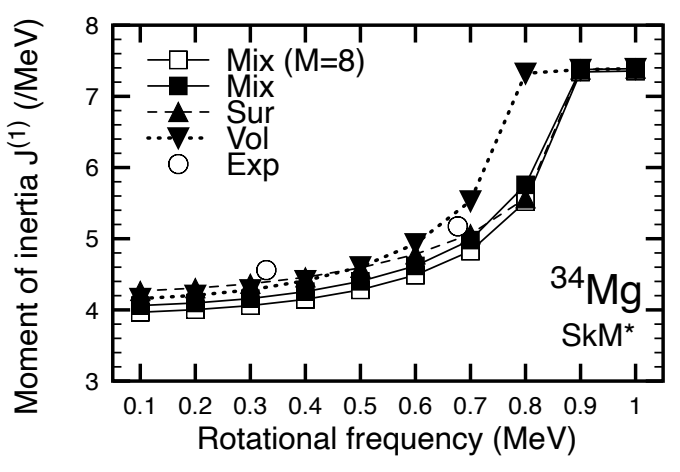

FIG. 2. Similar to Fig. 1 but for ${ }^{34} \mathrm{Mg}$. The results obtained by using several pairing interactions are displayed. The experimental data are obtained from Ref. [46].

the band crossing is also reasonably described. The $\mathrm{SkM}^{*}$ functional describes the alignment around $\omega_{\text {rot }}=0.35$ $\mathrm{MeV}$, however the level crossing is more gentle than in the case of SLy4.

\section{B. Neutron-rich $\mathrm{Mg}$ isotopes}

Then, I investigate the low-spin yrast states in the neutron-rich $\mathrm{Mg}$ isotopes. I use the $\mathrm{SkM}^{*}$ functional [41] and the so-called mixed-type pairing interaction with the strength $V_{0}=-295 \mathrm{MeV} \mathrm{fm}^{3}$ as in Refs. [28, 47], in which the low-frequency vibrations were investigated. The ground-state properties thus obtained are summarized in Table 1 of Ref. [28]. I found that the triaxiality is negligibly small: $\gamma<1^{\circ}$ in low spins. The chemical potential is a key quantity governing the spatial structure of quasiparticle wave functions: $-4.17,-3.26,-2.42,-1.61$ $\mathrm{MeV}$ for neutrons in ${ }^{34,36,38,40} \mathrm{Mg}$, respectively. When the two-basis method, in which the KSB Hamiltonian is diagonalized in a truncated single-particle basis obtained by solving the KS equation in the Cartesianmesh [8], is employed for such weakly bound nuclei, the single-particle scattering states enter the pairing window. Therefore, the convergence with respect to the number of basis states has to be carefully examined as the densities are spatially localized for $\lambda<0$. In the present case, however, the densities are always calculated to be localized because the full KSB Hamiltonian is directly diagonalized [12].

Figure 2 shows the calculated $\mathcal{J}^{(1)}$ of ${ }^{34} \mathrm{Mg}$, and compares with the experimental data [46]. The measured $R_{4 / 2}$ value is 3.06 , which is lower than that of the rigid rotor and close to the calculated value 2.96. The calculation reproduces well the slight increase in $\mathcal{J}^{(1)}$ due to the weakening of pairing. To see the effect of weak binding, the results obtained by using $M=8$ are also included in Fig. 2. The difference between the cases with $M=8$ and 12 is not very significant, which indicates that the spatial extension of neutrons is not important.
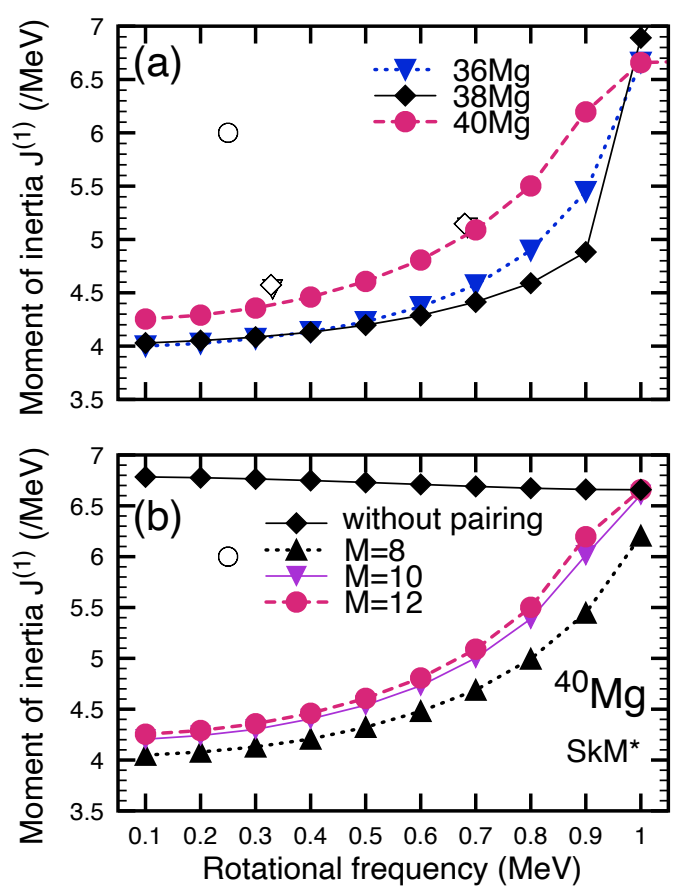

FIG. 3. (a) Similar to Fig. 2 but for ${ }^{36,38,40} \mathrm{Mg}$. The experimental data, denoted by open symbols, are taken from Refs. [20, 24]. (b) Calculated $\mathcal{J}^{(1)}$ for ${ }^{40} \mathrm{Mg}$ obtained with the box size of $M=8,10$, and 12 . Results without the pairing are also depicted.

It is noted that the calculated rms radius is $3.49 \mathrm{fm}$ and $3.14 \mathrm{fm}$ for neutrons and protons, respectively. That I obtained the converged results with a box size of $7-8 \mathrm{fm}$ is in accordance with the above example for ${ }^{172} \mathrm{Hf}$.

The role of the density dependence of the pairing interaction has been discussed in the study of the superdeformed states [48]: the density dependence results in a retarded alignment. I investigate here the density dependence of the pairing interaction. To this end, I use the volume- and surface-type pairing interactions. I determined the strength to keep the calculated pairing gap as obtained with the mixed pairing at $\omega_{\text {rot }}=0 \mathrm{MeV}$ : $V_{0}=-211$ and $-423 \mathrm{MeV} \mathrm{fm}^{3}$ for the volume and surface pairing, respectively. The pairing gap is defined by $\Delta^{q}=\int d \boldsymbol{r} \hat{h}^{q}(\boldsymbol{r}) \tilde{\rho}^{q *}(\boldsymbol{r}) / \int d \boldsymbol{r} \tilde{\rho}^{q *}(\boldsymbol{r})$. Notice that the protons are unpaired at $\omega_{\text {rot }}=0$. In low $\omega_{\text {rot }}$, the difference among three types of interaction is relatively small. However, the volume pairing gives a faster increase in $\mathcal{J}^{(1)}$ similarly to the finding in Ref. [48].

Next, I investigate the rotational property of ${ }^{36,38,40} \mathrm{Mg}$ located close to the drip line. Figure 3(a) displays the calculated $\mathcal{J}^{(1)}$ together with the experimental data [20, 24]. The calculated $\mathcal{J}^{(1)}$ for ${ }^{36,38} \mathrm{Mg}$ in low $\omega_{\text {rot }}$ is similar to the one for ${ }^{34} \mathrm{Mg}$, and smaller than that for ${ }^{40} \mathrm{Mg}$. This is consistent with the calculation in Ref. [28], where the moments of inertia were evaluated using the ThoulessValatin procedure in the framework of the Skyrme EDFbased QRPA [49]. A higher value for $\mathcal{J}^{(1)}$ of ${ }^{40} \mathrm{Mg}$ is 


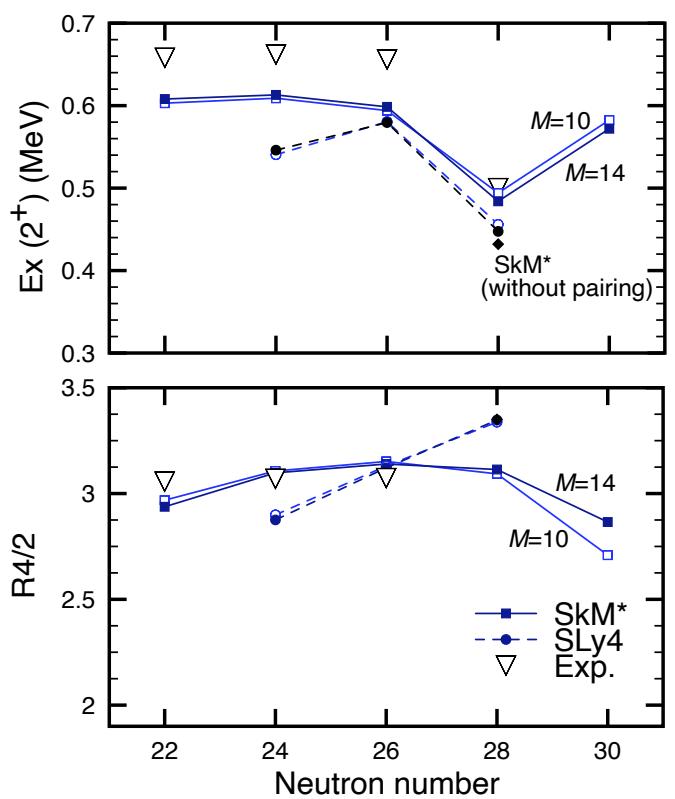

FIG. 4. Calculated $E\left(2^{+}\right)$(upper) and $R_{4 / 2}$ (lower) values with the $\mathrm{SkM}^{*}$ (squares) and SLy4 (circles) functionals. The results obtained by using $M=10$ and 14 are depicted by open and filled symbols, respectively. The result without the pairing is shown by a diamond for $\mathrm{SkM}^{*}$ at $N=28$. The experimental data are displayed by open triangles.

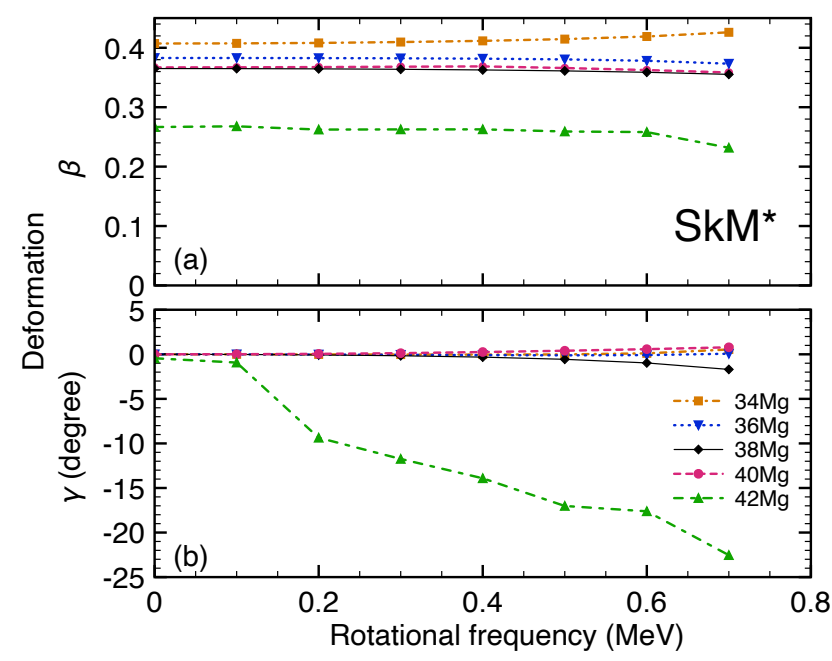

FIG. 5. Deformation parameters of protons in ${ }^{34,36,38,40} \mathrm{Mg}$.

partly because of smaller deformation than others; see Table 1 of Ref. [28]. Another reason is the weak-binding effect, as discussed below. The experimental data indicate that the pairing in ${ }^{36,38} \mathrm{Mg}$ would be weaker than the calculation because the measured $\mathcal{J}^{(1)}$ is slightly larger than the calculated one. Furthermore, the measurement shows a faster increase in $\mathcal{J}^{(1)}$ than the calculation for ${ }^{38} \mathrm{Mg}$; the calculation produces a stronger pairing correlation.
The present calculation fails to describe the rotational motion in ${ }^{40} \mathrm{Mg}$ : the calculated $\mathcal{J}^{(1)}$ is far below the measured value. One is then tempted to expect that the weak-binding effect shows up. In Fig. 3(b), I show the results obtained by varying the box size. With the increase in the box size, the calculated $\mathcal{J}^{(1)}$ increases. The calculation with $M=8$ does not show the convergence, while the difference between the results with $M=10$ and 12 becomes small. One cannot expect a further increase in $\mathcal{J}^{(1)}$ even enlarging the box size more. Notice that for the case of ${ }^{34} \mathrm{Mg}$, the results obtained with $M=8$ and 10 are not very different, as shown in Fig. 2. In this sense, the weak-binding effect appears in ${ }^{40} \mathrm{Mg}$; the weak binding of neutrons reduces the pairing correlation and enhances the moment of inertia. As an extreme case of weak pairing, I performed the calculation without the pairing, as displayed in Fig. 3(b). The resultant $\mathcal{J}^{(1)}$ is much larger than that obtained with pairing. As increasing the rotational frequency, the results with and without pairing become closer to each other. The observed $\mathcal{J}^{(1)}$ of $6.0 \mathrm{MeV}^{-1}$ is between these results and is relatively closer to the one obtained without pairing $\mathcal{J}^{(1)}=6.8 \mathrm{MeV}^{-1}$. As pointed out in Ref. [16], one has an oblate minimum in ${ }^{40} \mathrm{Mg}$. Indeed, I found the oblate solution with $\beta=0.16, \gamma=60^{\circ}$ though this is located higher in energy by $0.83 \mathrm{MeV}$. With this configuration, the calculated $\mathcal{J}^{(1)}$ is much lower: $1.9 \mathrm{MeV}^{-1}$.

To describe the isotopic dependence of low-spin states in the $\mathrm{Mg}$ isotopes, a key is the isospin dependence of the pairing correlation. In Ref. [50], it has been pointed out that the inclusion of the isospin dependence in the pairing EDF gives a nice reproduction of the pairing gaps in both stable and neutron-rich nuclei and in both symmetric nuclear matter and in neutron matter. Thus, I employ the optimal pairing EDF, the YSN functional [42] as above, in which the isovector density is introduced to describe pairing in nuclei in a wide mass region. Figure 4 shows the evaluated $E\left(2^{+}\right)$and $R_{4 / 2}$ values. Here, the spin $I$ is evaluated as $J_{z}^{2}=I(I+1)$.

An almost-constant $E\left(2^{+}\right)$and $R_{4 / 2}$ in $N=22-26$ is well described by using the $\mathrm{SkM}^{*}+\mathrm{YSN}$ functional. This model also describes well the decrease in energy at $N=28$ whereas this predicts the $R_{4 / 2}$ value keeps $\sim 3$. When the pairing is discarded, $E\left(2^{+}\right)$becomes much lower, and $R_{4 / 2}$ reaches 3.3 as depicted by a diamond. The results obtained by employing the SLy4+YSN functional are also shown in Fig. 4. The SLy4+YSN predicts that neutrons and protons are both unpaired in ${ }^{40} \mathrm{Mg}$. Thus, this gives a similar result to the $\mathrm{SkM}^{*}$ model without pairing. Whether the pairing of neutrons vanishes or not is discriminated by the $R_{4 / 2}$ value ${ }^{2}$.

When two neutrons are added, I find a further structural change. Comparing with ${ }^{40} \mathrm{Mg}$, the deformation

\footnotetext{
2 If the spin of the observed second excited state is $4^{+}$, the $R_{4 / 2}$ value is 2.34 [24].
} 
gets weaker by about $33 \%$, and the triaxiality emerges as shown in Fig. 5, where the sign of $\gamma$ is defined in the convention of Ref. [51]. The triaxiality develops as increasing the spin. The appearance of the triaxiality in the $N=30$ isotones has also been discussed in Ref. [52]. The pairing for protons shows up, and that for neutrons increases. Accordingly, $\mathcal{J}^{(1)}$ is reduced and thus $E\left(2^{+}\right)$ increases as shown in Fig. 4. The present calculation with $\mathrm{SkM}^{*}+$ YSN predicts that the irregularity in $E\left(2^{+}\right)$appears only at ${ }^{40} \mathrm{Mg}$. Because of the developed triaxiality at finite $\omega_{\text {rot }}$, the $R_{4 / 2}$ value deviates from that of the rigid rotor in ${ }^{42} \mathrm{Mg}$. It is noted that the SLy4 functional gives $\lambda^{\nu}>0$ for ${ }^{42} \mathrm{Mg}$.

A weak-binding effect is investigated by varying the box size and the role in the low-spin states is displayed in Fig. 4. The results obtained by using $M=10$ are compared with those obtained by using $M=14$. I found that the calculated $\mathcal{J}^{(1)}$ at $\omega_{\text {rot }}=0.1 \mathrm{MeV}$ changes by about $1.3 \%$ when the box size is varied for ${ }^{42} \mathrm{Mg}$. Since the chemical potential of neutrons is not very shallow, that is $-1.15 \mathrm{MeV}$ for the case of $\mathrm{SkM}^{*}$, a role of the spatial extension of neutrons is not visible in the $E\left(2^{+}\right)$ value. However, the spatial expansion varies depending on $\omega_{\text {rot }}$ as one finds that the $R_{4 / 2}$ value becomes smaller by $6 \%$ in enlarging the box size from $M=10$ to 14 . Investigation of not only the $2^{+}$state but higher-spin states in drip-line nuclei reveals unique roles of loosely bound neutrons. A triaxialy-deformed rotating nucleus is, for example, interesting future work to study [53].

\section{CONCLUSION}

To summarize, I have developed a numerical framework for a mean-field description of yrast states in nu- clei near the drip line in a nuclear EDF approach. To this end, I directly solved the coordinate-space cranked Skyrme-KSB equation in 3D mesh, with the continuum states being discretized in a box. The present framework reproduces the low-spin states and band crossing in a medium-heavy deformed nucleus.

The low-spin states in ${ }^{34} \mathrm{Mg}$ are well described by using the density-dependent pairing interaction. With the increase in the neutron number, the calculation overestimates the pairing correlation, thus leading to the underestimation of the moments of inertia. Employing the optimal pairing-EDF constructed to describe neutron-rich nuclei, I have found that the appearance of the significantly low $I^{\pi}=2^{+}$state in ${ }^{40} \mathrm{Mg}$ is mainly due to the suppression of pairing. A systematic study of the $2^{+}$ state in neutron-rich nuclei thus provides a constraint on the global pairing EDF. In ${ }^{42} \mathrm{Mg}$, the $2_{1}^{+}$state appears higher in energy than in ${ }^{40} \mathrm{Mg}$, and the $R_{4 / 2}$ value decreases due to the structure change, where the triaxial deformation emerges.

\section{ACKNOWLEDGMENTS}

This work was supported by the JSPS KAKENHI (Grants No. JP19K03824 and No. JP19K03872). The numerical calculations were performed on the computing facilities at the Yukawa Institute for Theoretical Physics, Kyoto University, and at the Research Center for Nuclear Physics, Osaka University.
[1] T. Otsuka, A. Gade, O. Sorlin, T. Suzuki, and Y. Utsuno, Evolution of shell structure in exotic nuclei, Rev. Mod. Phys. 92, 015002 (2020).

[2] A. Gade, Excitation energies in neutron-rich rare isotopes as indicators of changing shell structure, Eur. Phys. J. A 51, 118 (2015).

[3] A. Bohr and B. Mottelson, Nuclear Structure: Volume II, Nuclear Deformations (Benjamin, Reading, MA, 1975).

[4] M. J. A. de Voigt, J. Dudek, and Z. Szymański, Highspin phenomena in atomic nuclei, Rev. Mod. Phys. 55, 949 (1983).

[5] R. Bengtsson, S. Frauendorf, and F.-R. May, Quasiparticle levels in rotating rare earth nuclei: A cranked shell-model dictionary, At. Data Nucl. Data Tab. 35, 15 (1986).

[6] J. D. Garrett, G. B. Hagemann, and B. Herskind, Recent nuclear structure studies in rapidly rotating nuclei, Ann. Rev. Nucl. Part. Sci. 36, 419 (1986), https://doi.org/10.1146/annurev.ns.36.120186.002223.

[7] W. Satuła and R. A. Wyss, Mean-field description of high-spin states, Rep. Prog. Phys. 68, 131 (2004).
[8] M. Bender, P.-H. Heenen, and P.-G. Reinhard, Selfconsistent mean-field models for nuclear structure, Rev. Mod. Phys. 75, 121 (2003).

[9] D. Vretenar, A. Afanasjev, G. Lalazissis, and P. Ring, Relativistic hartree-bogoliubov theory: static and dynamic aspects of exotic nuclear structure, Phys. Rep. 409, 101 (2005).

[10] L. M. Robledo, T. R. Rodríguez, and R. R. RodríguezGuzmán, Mean field and beyond description of nuclear structure with the gogny force: a review, J. Phys. G: Nucl. Part. Phys. 46, 013001 (2018).

[11] M. Yamagami and Y. R. Shimizu, Pairing effects for rotational excitations unique to neutron-rich nuclei, Phys. Rev. C 77, 064319 (2008).

[12] J. Dobaczewski, H. Flocard, and J. Treiner, HartreeFock-Bogolyubov description of nuclei near the neutrondrip line, Nucl. Phys. A 422, 103 (1984).

[13] K. Hara and Y. Sun, Projected Shell Model and HighSpin Spectroscopy, Int. J. Mod. Phys. E 4, 637 (1995).

[14] T. Nakatsukasa, K. Matsuyanagi, M. Matsuo, and K. Yabana, Time-dependent density-functional description of 
nuclear dynamics, Rev. Mod. Phys. 88, 045004 (2016), arXiv:1606.04717.

[15] O. Sorlin and M.-G. Porquet, Nuclear magic numbers: New features far from stability, Prog. Part. Nucl. Phys. 61, 602 (2008).

[16] J. Terasaki, H. Flocard, P.-H. Heenen, and P. Bonche, Deformation of nuclei close to the two-neutron drip line in the Mg region, Nucl. Phys. A 621, 706 (1997).

[17] M. Kimura and H. Horiuchi, Breaking of the Neutron Magic Number $N=20$ in ${ }^{32} \mathrm{Mg}$ and ${ }^{30} \mathrm{Ne}$ and Its Possible Relation to the Cluster Structure, Prog. Theor. Phys. 107, 33 (2002), https://academic.oup.com/ptp/articlepdf/107/1/33/5213174/107-1-33.pdf.

[18] R. Rodríguez-Guzmán, J. Egido, and L. Robledo, Correlations beyond the mean field in magnesium isotopes: angular momentum projection and configuration mixing, Nucl. Phys. A 709, 201 (2002).

[19] N. Hinohara, K. Sato, K. Yoshida, T. Nakatsukasa, M. Matsuo, and K. Matsuyanagi, Shape fluctuations in the ground and excited $0^{+}$states of ${ }^{30,32,34} \mathrm{Mg}$, Phys. Rev. C 84, 061302 (2011).

[20] P. Doornenbal, H. Scheit, S. Takeuchi, N. Aoi, K. Li, M. Matsushita, D. Steppenbeck, H. Wang, H. Baba, H. Crawford, C. R. Hoffman, R. Hughes, E. Ideguchi, N. Kobayashi, Y. Kondo, J. Lee, S. Michimasa, T. Motobayashi, H. Sakurai, M. Takechi, Y. Togano, R. Winkler, and K. Yoneda, In-Beam $\gamma$-Ray Spectroscopy of ${ }^{34,36,38} \mathrm{Mg}$ : Merging the $N=20$ and $N=28$ Shell Quenching, Phys. Rev. Lett. 111, 212502 (2013).

[21] M. Shimada, S. Watanabe, S. Tagami, T. Matsumoto, Y. R. Shimizu, and M. Yahiro, Simultaneous analysis of matter radii, transition probabilities, and excitation energies of $\mathrm{Mg}$ isotopes by angular-momentumprojected configuration-mixing calculations, Phys. Rev. C 93, 064314 (2016), arXiv:1605.08585 [nucl-th].

[22] T. R. Rodríguez, Precise description of nuclear spectra with Gogny energy density functional methods, Eur. Phys. J. A 52, 190 (2016).

[23] J. M. Yao, H. Mei, H. Chen, J. Meng, P. Ring, and D. Vretenar, Configuration mixing of angular-momentum projected triaxial relativistic mean-field wave functions. II. Microscopic analysis of low-lying states in magnesium isotopes, Phys. Rev. C 83, 014308 (2011), arXiv:1006.1400 [nucl-th].

[24] H. L. Crawford, P. Fallon, A. O. Macchiavelli, P. Doornenbal, N. Aoi, F. Browne, C. M. Campbell, S. Chen, R. M. Clark, M. L. Cortés, M. Cromaz, E. Ideguchi, M. D. Jones, R. Kanungo, M. MacCormick, S. Momiyama, I. Murray, M. Niikura, S. Paschalis, M. Petri, H. Sakurai, M. Salathe, P. Schrock, D. Steppenbeck, S. Takeuchi, Y. K. Tanaka, R. Taniuchi, H. Wang, and K. Wimmer, First Spectroscopy of the Near Drip-line Nucleus ${ }^{40} \mathrm{Mg}$, Phys. Rev. Lett. 122, 052501 (2019).

[25] K. Yoshida, M. Yamagami, and K. Matsuyanagi, Pairing and continuum effects on low-frequency quadrupole vibrations in deformed $\mathrm{Mg}$ isotopes close to the neutron drip line, Nucl. Phys. A 779, 99 (2006), arXiv:nuclth/0605073.

[26] K. Yoshida, M. Yamagami, and K. Matsuyanagi, Dynamic pairing effects on low-frequency modes of excitation in deformed $\mathrm{Mg}$ isotopes close to the neutron drip line, Phys. Scr. T 125, 45 (2006), arXiv:nucl-th/0507047.

[27] K. Yoshida and M. Yamagami, Low-frequency $K^{\pi}=0^{+}$ modes in deformed neutron-rich nuclei: Pairing- and $\beta$ - vibrational modes of neutron, Phys. Rev. C 77, 044312 (2008), arXiv:0802.2341 [nucl-th].

[28] K. Yoshida, Skyrme-QRPA calculations for low-lying excitation modes in deformed neutron-rich nuclei, Eur. Phys. J. A 42, 583 (2009), arXiv:0902.3053 [nucl-th].

[29] M. Yamagami, Pairing effect on $K^{\pi}=0^{+}$quadrupole excitations in neutron-rich $\mathrm{Mg}$ isotopes studied by Skyrme quasiparticle random-phase approximation calculations in wave-number space, Phys. Rev. C 100, 054302 (2019).

[30] S. Jin, A. Bulgac, K. Roche, and G. Wlazłowski, Coordinate-space solver for superfluid many-fermion systems with the shifted conjugate-orthogonal conjugategradient method, Phys. Rev. C 95, 044302 (2017), arXiv:1608.03711 [nucl-th].

[31] Y. Kashiwaba and T. Nakatsukasa, Coordinate-space solver for finite-temperature Hartree-Fock-Bogoliubov calculations using the shifted Krylov method, Phys. Rev. C 101, 045804 (2020), arXiv:2001.00500 [nucl-th].

[32] S. Jin, K. J. Roche, I. Stetcu, I. Abdurrahman, and A. Bulgac, The LISE package: Solvers for static and time-dependent superfluid local density approximation equations in three dimensions, Comp. Phys. Commun. 269, 108130 (2021).

[33] S. Sakai, K. Yoshida, and M. Matsuo, Signature dependent triaxiality for shape evolution from superdeformation in rapidly rotating ${ }^{40} \mathrm{Ca}$ and ${ }^{41} \mathrm{Ca}$, Prog. Theor. Exp. Phys. 2020, 063 D02 (2020), arXiv:2003.06081 [nucl-th].

[34] K. Yoshida, Competing shell effect of protons and neutrons for superdeformation in ${ }^{60} \mathrm{Zn},{ }^{62} \mathrm{Zn}$, and ${ }^{64} \mathrm{Ge}$, (2021), arXiv:2108.12130 [nucl-th].

[35] P. Bonche, H. Flocard, and P. Heenen, Self-consistent calculation of nuclear rotations: The complete yrast line of ${ }^{24} \mathrm{Mg}$, Nucl. Phys. A 467, 115 (1987).

[36] H. Ogasawara, K. Yoshida, M. Yamagami, S. Mizutori, and K. Matsuyanagi, Rotational Frequency Dependence of Octupole Vibrations on Superdeformed States in ${ }^{40} \mathrm{Ca}$, Prog. Theor. Phys. 121, 357 (2009).

[37] ScaLAPACK - Scalable Linear Algebra PACKage, http: //www.netlib.org/scalapack/.

[38] A. Baran, A. Bulgac, M. M. Forbes, G. Hagen, W. Nazarewicz, N. Schunck, and M. V. Stoitsov, Broyden's method in nuclear structure calculations, Phys. Rev. C 78, 014318 (2008).

[39] National Nuclear Data Center, "Evaluated Nuclear Structure Data File", https://www.nndc.bnl.gov/ ensdf/.

[40] E. Chabanat, P. Bonche, P. Haensel, J. Meyer, and R. Schaeffer, A Skyrme parametrization from subnuclear to neutron star densities. 2. Nuclei far from stablities, Nucl. Phys. A 635, 231 (1998), [Erratum: Nucl.Phys.A 643, 441-441 (1998)].

[41] J. Bartel, P. Quentin, M. Brack, C. Guet, and H.-B. Håkansson, Towards a better parametrisation of Skyrmelike effective forces: A critical study of the SkM force, Nucl. Phys. A 386, 79 (1982).

[42] M. Yamagami, Y. R. Shimizu, and T. Nakatsukasa, Optimal pair density functional for description of nuclei with large neutron excess, Phys. Rev. C 80, 064301 (2009), arXiv:0812.3197 [nucl-th].

[43] K. Davies, H. Flocard, S. Krieger, and M. Weiss, Application of the imaginary time step method to the solution of the static Hartree-Fock problem, Nucl. Phys. A 342, 111 (1980).

[44] P. Bonche, H. Flocard, P. Heenen, S. Krieger, and 
M. Weiss, Self-consistent study of triaxial deformations: Application to the isotopes of $\mathrm{Kr}, \mathrm{Sr}, \mathrm{Zr}$ and Mo, Nucl. Phys. A 443, 39 (1985).

[45] N. Tajima, Hartree-Fock + BCS approach to unstable nuclei with the Skyrme force, Prog. Theor. Phys. Suppl. 142, 265 (2001), arXiv:nucl-th/0307085.

[46] S. Michimasa, Y. Yanagisawa, K. Inafuku, N. Aoi, Z. Elekes, Z. Fülöp, Y. Ichikawa, N. Iwasa, K. Kurita, M. Kurokawa, T. Machida, T. Motobayashi, T. Nakamura, T. Nakabayashi, M. Notani, H. J. Ong, T. K. Onishi, H. Otsu, H. Sakurai, M. Shinohara, T. Sumikama, S. Takeuchi, K. Tanaka, Y. Togano, K. Yamada, M. Yamaguchi, and K. Yoneda, Quadrupole collectivity in islandof-inversion nuclei ${ }^{28,30} \mathrm{Ne}$ and ${ }^{34,36} \mathrm{Mg}$, Phys. Rev. C 89, 054307 (2014).

[47] K. Yoshida, Pygmy dipole mode in deformed neutronrich $\mathrm{Mg}$ isotopes close to the drip line, Phys. Rev. C 80, 044324 (2009), arXiv:0908.3085 [nucl-th].

[48] J. Terasaki, P.-H. Heenen, P. Bonche, J. Dobaczewski, and H. Flocard, Superdeformed rotational bands with density dependent pairing interactions, Nucl. Phys. A 593, 1 (1995).

[49] K. Yoshida and N. Van Giai, Deformed quasiparticlerandom-phase approximation for neutron-rich nuclei using the Skyrme energy density functional, Phys. Rev. C 78, 064316 (2008), arXiv:0809.0169 [nucl-th].

[50] J. Margueron, H. Sagawa, and K. Hagino, Effective pairing interactions with isospin density dependence, Phys. Rev. C 77, 054309 (2008), arXiv:0712.3644 [nucl-th].

[51] S. G. Nilsson and I. Ragnarsson, Shapes and shells in nuclear structure (Cambridge university press, Cambridge, 1995).

[52] Y. Suzuki and M. Kimura, Triaxial deformation and the disappearance of the $N=28$ shell gap, Phys. Rev. C 104, 024327 (2021), arXiv:2103.06086 [nucl-th].

[53] K. Uzawa, K. Hagino, and K. Yoshida, Role of triaxiality in deformed halo nuclei, Phys. Rev. C 104, L011303 (2021), arXiv:2104.02946 [nucl-th]. 